\title{
Nails in systemic disease
}

\author{
Authors: Charlotte E Gollins ${ }^{A}$ and David de Berker ${ }^{B}$
}

A change in colour, size, shape or texture of finger- and toenails can be an indicator of underlying systemic disease. An appreciation of these nail signs, and an ability to interpret them when found, can help guide diagnosis and management of a general medical patient. This article discusses some common, and some more rare, nail changes associated with systemic disease.

\section{Introduction}

Examination of nails is a skill that, although emphasised when revising for general medical exams, can be overlooked in dayto-day practice. The value of noticing, understanding and interpreting nail changes can positively add to clinical practice as these signs can provide valuable clues to a diagnosis.

Here we present a brief overview of selected common and rarer nail abnormalities associated with systemic conditions, as well as a limited explanation of the pathophysiology of some of the changes.

\section{Anatomy of the nail unit}

The nail unit (Fig 1) is an epithelial skin appendage composed of the hardened nail plate surrounded by specialised epithelial surfaces that contribute to its growth and maintenance. The nail plate is formed of keratinised epithelial cells. It is produced within the nail matrix and grows distally at a rate of approximately $3.5 \mathrm{~mm}$ and $1.6 \mathrm{~mm}$ per month in fingernails and toenails, respectively. ${ }^{2}$ The nail matrix can be macroscopically seen as the lunula. As the matrix is the site of production of the nail plate, factors which impact it (for example, through direct pressure or inflammation) will disrupt this process and lead to a change in shape, size, texture and colour of the nail plate as it grows. ${ }^{3}$ This is often the process through which systemic disease leads to clinical nail changes.

\section{Cardiac disorders}

\section{Bacterial endocarditis}

Splinter haemorrhages (Fig 2) are small, linear subungual structures, normally red, purple or brown in colour, most frequently

Authors: Adermatology registrar, University Hospitals Bristol and Weston NHS Foundation Trust, Bristol, UK; ${ }^{B}$ Consultant dermatologist, University Hospitals Bristol and Weston NHS Foundation Trust, Bristol, UK
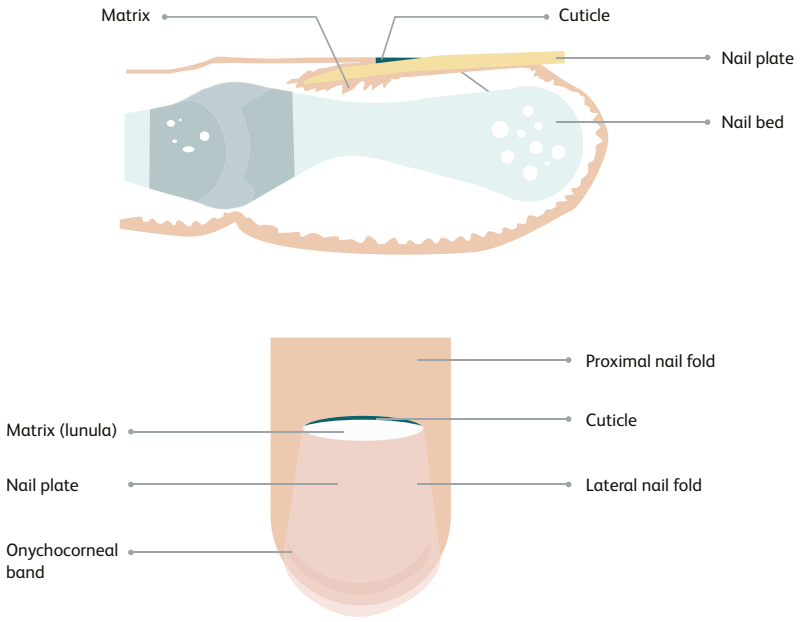

Fig 1. Anatomy of the nail plate.

located in the distal third of the nail plate. They are caused by damage to capillaries within the nail bed, which have a longitudinal orientation, leading to their linear appearance. In the case of bacterial endocarditis, this damage is likely to be caused by platelet microthrombi within these vessels. Splinter haemorrhages are, however, also seen in many other conditions (for example vasculitis) and most commonly due to trauma. ${ }^{4}$

\section{Key points}

Damage to the nail matrix will lead to abnormalities in the nail plate.

Changes that affect all 20 nails are likely to be due to an underlying systemic illness, drug or genetic mutation.

Simple clubbing of nails is most commonly caused by an acquired thoracic disease.

Recurrent nail infections and chronic paronychia can be a sign of underlying immunosuppression.

The nails are a key site of abnormalities in connective tissue diseases and vasculitis.

KEYWORDS: nail, clubbing, Beau's, matrix, koilonychia

DOI: 10.7861/clinmed.2021-0243 


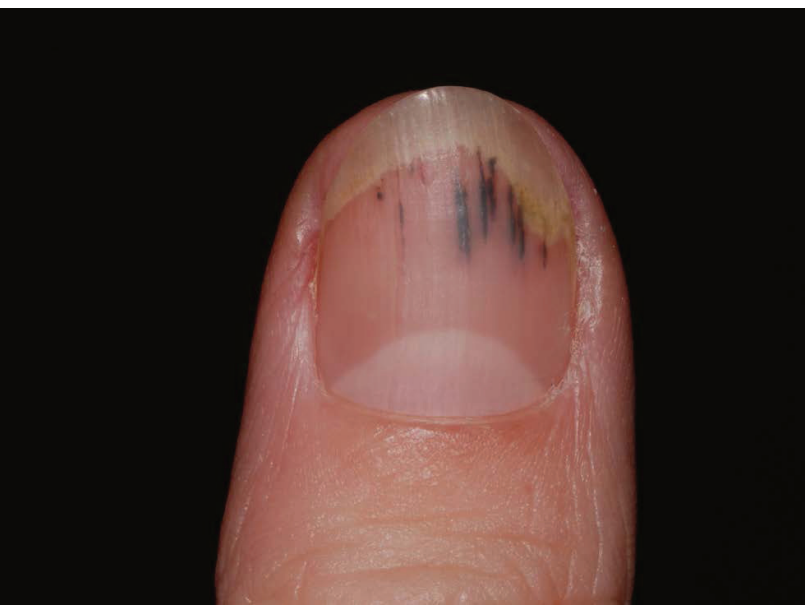

Fig 2. Splinter haemorrhages.

Further signs of subacute bacterial endocarditis are Osler's nodes and Janeway lesions. Osler's nodes are tender red nodules which develop in the finger pulps and along both the proximal and lateral nail folds. Janeway lesions are more distal, non-tender nodules affecting the palmoplantar surfaces. Both are likely to be caused by septic emboli.

\section{Congenital heart disease}

Clubbing is defined as an increase in the angle between the nail plate and proximal nail fold (Lovibond's angle) and can be most easily clinically ascertained by placing the dorsal surface of symmetrical opposing fingers together, and assessing for the obliteration of the diamond-shaped window between the fingers. Clinically, the first sign is of a sponginess at the base of the nail, allowing a slight movement of the nail plate when the proximal nail fold is pressed. This is proposed to be caused by fibrovascular hyperplasia of the tissue between the matrix and the underlying periosteum. Following this, the nail plate develops what appears to be an increased curvature. Several pathophysiological mechanisms have been suggested, including locally increased blood flow and proliferation of fibroblasts secondary to vascular endothelial growth factor. Associated with the congenital cardiac defect, the right-left vascular shunt leads to cyanosis visible in the colour of the nail bed and peripheries which distinguishes it from clubbing due to other causes.

Clubbing occurs in many other conditions, with $80 \%$ of simple clubbing having an acquired thoracic cause including lung cancer, bronchiectasis and cystic fibrosis, with $5 \%$ due to acquired gastrointestinal conditions including oesophageal and gastric malignancies and inflammatory bowel disease. ${ }^{5}$

\section{Gastrointestinal and hepatic disorders}

\section{Poisoning}

Systemic poisoning is recognised as giving rise to a transverse white band in the nails referred to as Mees' lines (Fig 3). The classic agent is arsenic or thallium, reported in forensic literature. Chemotherapy can produce a similar effect, most commonly in the treatment of lymphomas where cycles of treatment may

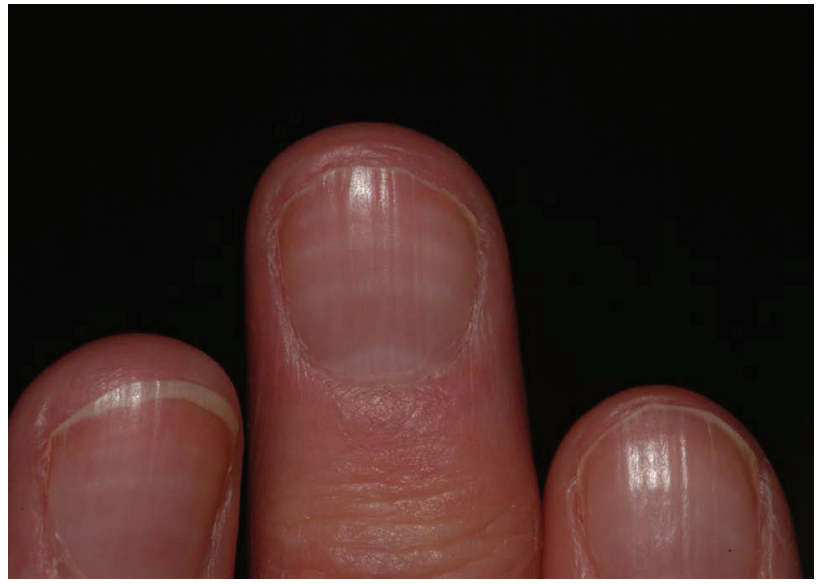

Fig 3. Mees' lines.

create a series of transverse white lines corresponding to the chronology.

\section{Gastrointestinal malignancy}

Upper gastrointestinal cancer can be associated with subungual hyperkeratosis and additional keratosis of the palms and acral skin in a presentation termed acrokeratosis paraneoplastica (Bazex syndrome). ${ }^{6}$

\section{Cirrhosis}

'Terry's nails' were first described by Terry in 1954, when he studied the nails of patients with liver cirrhosis. ${ }^{7}$ He found that $82 \%$ had an opaque white change to almost the entire nail bed, obscuring the lunula, leaving a small pink 1-2 mm band at the distal end of the nail. In addition to cirrhosis, 'Terry's nails' are associated with congestive heart failure and adult-onset diabetes, with this sign more likely to indicate systemic disease when found in younger patients. ${ }^{8}$

\section{Haematological disorders}

\section{Anaemia}

Nail bed pallor is seen in patients with anaemia and can be used as a diagnostic tool. Classically koilonychia (spoon shaped nails) has been associated with iron deficiency anaemia, and has been shown to improve with iron supplementation. ${ }^{9}$ With koilonychia, the nails are concave, with everted distal and lateral edges, giving a spoon-like appearance. The prevalence of koilonychia in healthy children is not associated with anaemia.

Koilonychia (Fig 4) can also be due to local trauma and skin diseases where there is nail thinning with concavity as a secondary mechanical response.

\section{Renal disorders}

\section{Renal failure}

'Half and half nails' are a sign similar to Terry's nails, and the distinction between the two can sometimes be difficult to 


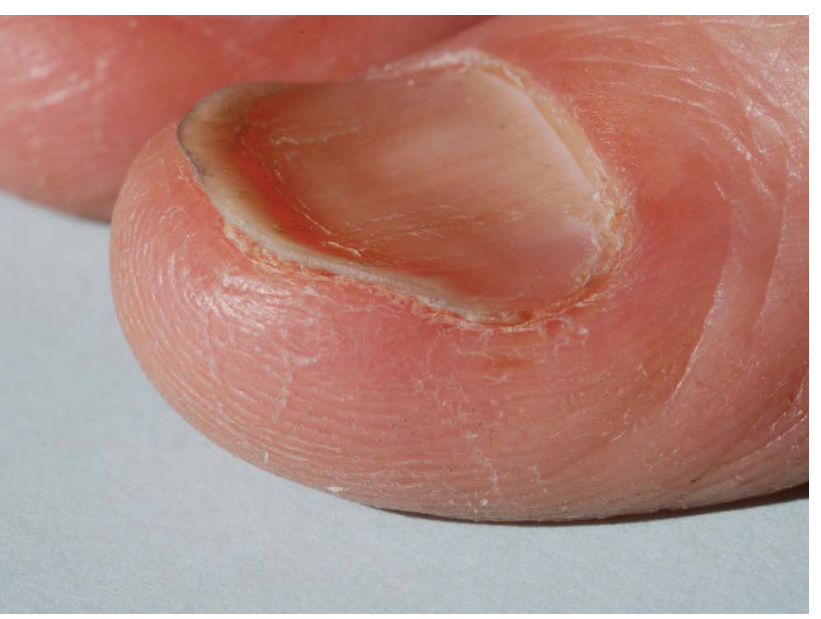

Fig 4. Koilonychia.

determine. The nails again have proximal white ground-glass changes, but the distal pink band is larger than in Terry's nails ( $>20 \%$ nail bed surface area) and can be red or brown in colour.

\section{Nail-patella syndrome}

Onycho-osteodysplasia (NPS) is a rare autosomal dominant condition caused by a mutation in the $L M X 1 B$ gene..$^{10}$ This syndrome presents with nail hypoplasia and atrophy, as well as distinctive triangular lunulae. Patients have hypoplastic patellae and may also have nephropathy and glaucoma. Recognition of the nail changes allow for appropriate screening for renal disease.

\section{Infectious diseases}

\section{HIV}

Untreated HIV or AIDs can present with nail infections; bacterial, viral and fungal. Proximal white onychomycosis is a pattern of fungal nail infection that is rarely seen without a diagnosis of immunosuppression, where HIV is an example; trichophyton rubrum is commonly the underlying pathogen. Candida species can also lead to acute and chronic onychomycosis and paronychia (inflammation of the periungual skin). ${ }^{11}$ Nail bed squamous cell carcinoma in younger patients can occur with HIV and is associated with human papillomavirus infection.

\section{Respiratory disorders}

\section{Smoking}

Superficial yellow-brown staining of the nail plate is commonly observed in smokers. If a patient quits suddenly, there will be a sharp demarcation in colour as the nail grows out, otherwise known as 'quitter's nail'.

\section{Yellow nail syndrome}

This disorder (Fig 5) is characterised by a triad of thick yellow nails (can affect all 20 nails), lymphoedema and respiratory tract involvement. Chronic sinusitis, pleural effusions, chronic cough and bronchiectasis are seen, along with lower leg oedema. The

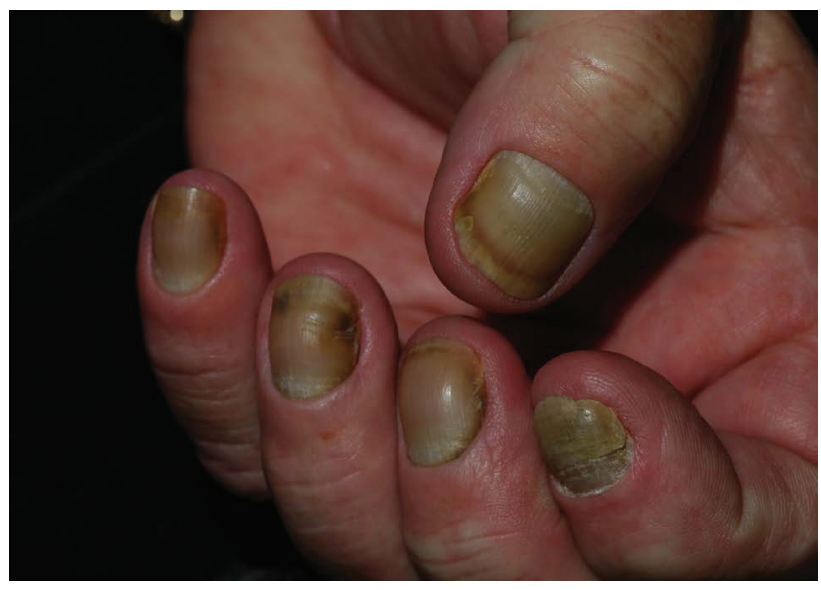

Fig 5. Yellow nail syndrome.

nail changes can precede respiratory manifestations by years and are thought to be due to very slow growth of the nails. Treatment is multifactorial and based on addressing focal features of inflammation or in-growing and nail shedding. Systemic treatments include vitamin E and itraconazole, but have a poor evidence base. ${ }^{12}$

\section{Rheumatological disorders}

\section{Systemic lupus erythematosus}

Various nail changes have been described in patients with systemic lupus erythematosus (SLE), including thinning of the nail plate, subungual hyperkeratosis, transverse leukonychia, pitting and onychorrhexis. None of these changes, however, is specific to SLE..$^{13}$ An informative site of nail change in connective tissue disease is the proximal nail fold, which can develop erythema, telangiectasia and nail fold infarcts. Capillaroscopy allows more detailed assessment of the nail fold capillaries, which in SLE have a meandering tortuous pattern. This is in comparison to scleroderma and dermatomyositis, in which the capillaries appear more disorganised and twisted..$^{14}$

\section{Systemic sclerosis}

Along with the proximal nail fold changes described earlier, periungual haemorrhages and ulceration occur in systemic sclerosis. ${ }^{14}$ Nail fold bleeding is significantly more common in patients with systemic sclerosis compared with other connective tissue diseases. The cuticles can be ragged, and nail plates can be thinned or develop onychorrhexis due to impaired peripheral circulation.

\section{Small vessel vasculitis}

Bywaters described small nail fold and finger pulp petechiae secondary to necrotising vasculitis in rheumatoid arthritis (Bywaters lesions). ${ }^{15}$ Nail fold infarcts and splinter haemorrhages are other lesions seen in small vessel vasculitides, either primary or secondary to a connective tissue disease. In more severe disease, haemorrhagic bullae and gangrene can form. 


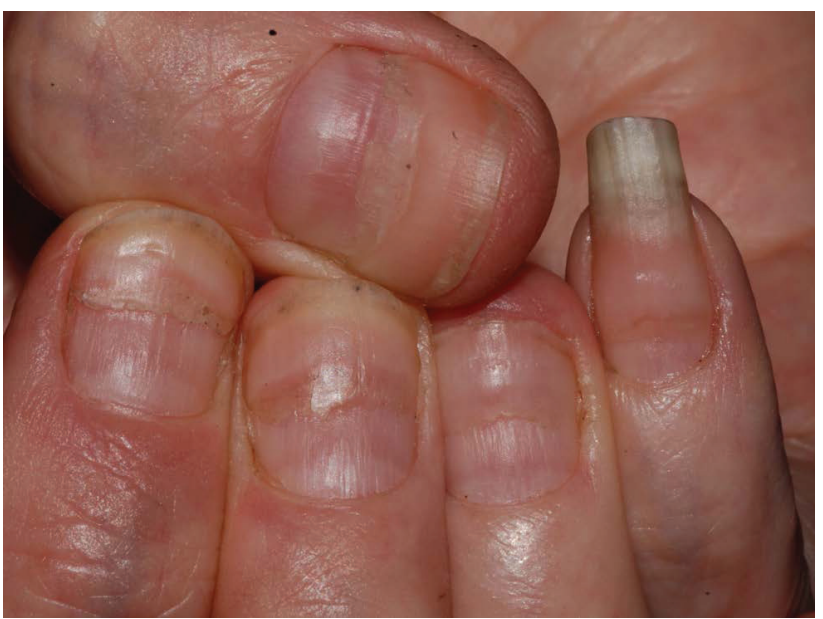

Fig 6. Beau's lines.

\section{General systemic illness}

\section{Beau's lines}

Transverse groves in the nail plate occur at a time of trauma or severe systemic illness, due to a temporary reduction in activity of the nail matrix (Fig 6). They may occur in all nails simultaneously but to different degrees, or be apparent in just a few. Profound or prolonged systemic upset may lead to complete division of the nail plate, but nail shedding will only arise if the adherence to the nail bed is lost (onychomadesis).

\section{Conclusion}

Although many nail signs are not pathognomonic for diseases, and can be seen in various systemic illnesses, they can be useful to build an overall picture of a patient's health. In some cases, they can directly contribute to diagnosis. We propose that closer inspection of the nails in general medical patients should be undertaken as part of the standard general medical examination.

\section{References}

1 Haneke E. Surgical anatomy of the nail apparatus. Dermatol Clin 2006:3:291-6.

2 Yaemsiri S, Hou N, Slining MM et al. Growth rate of human fingernails and toenails in healthy American young adults. J Eur Acad Dermatol Venereol 2010;24:420-3.

3 Baran R, de Berker DAR, Holzberg $M$ et al. Diseases of the nails and their management, 4th edn. Wiley-Blackwell, 2012.

4 Fanning WL, Aronson M. Osler node, Janeway lesions and splinter haemorrhages. Arch Dermatol 1977;133:648-9.

5 Stone OJ. Spoon nails and clubbing. Cutis 1975;16:235-41.

6 Schadt C. Cutaneous signs of malignancy. Semin Oncol 2016;43: 341-6.

7 Terry RB. White nails in hepatic cirrhosis. Lancet 1954;1:757-9.

8 Holzberg M, Walker HK. Terry's nails: revised definitions and new correlations. Lancet 1984;1:896-9.

9 Hogan GR, Jones B. The relationship of koilonychia and iron deficiency in infants. J Paediatr 1970;77:1054.

10 MacIntosh I, Dreyer SD, Clough MV et al. Mutation analysis of LMX1B gene in nail-patella syndrome patients. Am J Hum Genet 1998:63:1651-8.

11 Daniel CR, Norton LA, Scher RK. The spectrum of nail diseases in patients with HIV infection. J Am Acad Dermatol 1992;27:93-7.

12 Vignes S, Baran R. Yellow nail syndrome. Orphanet J Rare Dis 2017 12:1-10.

13 Sherber N, Wigley FM, Scher RK. Autoimmune disorders: nail signs and therapeutic approaches. Dermatol Ther 2007;20:17-30.

14 Maeda M, Kachi H, Takagi H. Haemorrhagic patterns of the cuticles distal to the proximal nail folds in the fingers of patients with systemic scleroderma. Eur ] Dermatol 1997;7:191-6.

15 Bywaters EG, Scott JT. Peripheral vascular obstruction in rheumatoid arthritis and its relationship to other vascular lesions. Ann Rheum Dis 1957;16:84-103.

Address for correspondence: Dr David de Berker, Dermatology Department, Bristol Royal Infirmary, Marlborough Street, Bristol, Avon BS2 8HW, UK.

Email: david.deberker@uhbw.nhs.uk 